\section{Mit Technik gegen den demografischen Wandel} Bericht zum 6. Deutschen AAL-Kongress

Berlin, 22.-23. Januar 2013

\section{von Manuel Dietrich und Kolja Bopp, ITAS}

Der demografische Wandel stellt unsere Gesellschaft vor enorme Herausforderungen. Aus diesem Grund setzt man hohe Erwartungen in technologische Entwicklungen, z. B. in den Einsatz von Servicerobotern für die Pflege bedürftiger Menschen. Auf dem 6. Deutschen Ambient-Assistant-Living-Kongress mit dem Titel „Lebensqualität im Wandel von Demografie und Technik" diskutierten ca. 500 Teilnehmer in insgesamt 21 Sessions, zumeist aus der Sicht einer anwendungsnahen Technikforschung, Sozialforschung zur Akzeptanz sowie einer Industrieperspektive. Bereits in der Einführung zum Kongress stellte der Staatsekretär im BMBF, Thomas Rachel, die Stoßrichtung des Kongresses vor: Der demografische Wandel sei eine große Herausforderung, so seine Prämisse, und zur Problembewältigung werde v. a. auf technologische Entwicklungen gesetzt. Fragen, die aus Sicht der Technikfolgenabschätzung zu stellen wären, etwa nach dem Potenzial neuer sozialer Arrangements oder dem Zusammenhang von technischen und sozialen Innovationen, gehörten nur am Rande ins Spektrum der Veranstaltung. Auch wurde der demografische Wandel selbst nicht hinterfragt. Dennoch war der Kongress dazu geeignet, aufgrund seiner breiten, praxisnahen Ausrichtung ein adäquates Abbild aktueller technischer Entwicklungen mit Blick auf Einsatzszenarios jetzt und in naher $\mathrm{Zu}$ kunft zu ermöglichen.

„Ambient Assisted Living“ (AAL) bezieht sich auf technische Assistenzsysteme im häuslichen Umfeld. Das Ziel ist, älteren Menschen länger ein selbstbestimmtes Leben in vertrauter Umgebung zu ermöglichen und damit auch Kosten für Pflege und Betreuung zu verringern. Die klassische AAL wird als eine im Hintergrund arbeitende, unauffällig unterstützende Technik gedacht. Szenarien sind dabei die Überwachung der Wohnung und des Menschen, z. B. durch intelligente Alarmsysteme, zentrale Steuerung vernetzter Hauselektronik oder Monitoring des
Gesundheitszustandes sowie automatische Erinnerungshilfen. Neuerdings werden auch mobile Robotersysteme für die AAL immer interessanter, da man sich dadurch eine zusätzliche physische Unterstützung (z. B. Hol- und Bringdienste) älterer Menschen erwartet.

\section{Klassische AAL}

In vielen Projekten sind Tablet-PCs mit Touchscreen ein wichtiger Bestandteil des Assistenzsystems. Sie ermöglichen Videotelefonie, dienen als Haustüröffner mit Livebild, werden zur Steuerung der Wohnungsüberwachung benutzt oder erinnern an die Medikamenteneinnahme. Meist wird etablierte Technik genutzt, die an die Bedürfnisse älterer Menschen angepasst wurde. Das Projekt „Länger selbstbestimmt Leben“ (Melina Frenken, Universität Oldenburg) versucht weitgehend auf teure Technik zu verzichten und von den Bedürfnissen der Zielgruppe auszugehen. Dazu wurde ein Workshop mit Teilnehmern eines Volkshochschulkurses „Leben im Alter" abgehalten, um alltägliche Probleme zu ermitteln. Eines der Probleme sei die Angst, beim Verlassen der Wohnung vergessen zu haben, das Bügeleisen oder den Herd auszuschalten. Ein „Alles-aus-Knopf" an der Haustür sei die einfachste Lösung, so die Referentin.

Andreas Hein (Universität Oldenburg) erwartet mehr Sicherheit für alleinstehende Personen durch das sog. Aktivitätsmonitoring. Dabei erfassen Sensoren die Aktivität einer Person, woraufhin Angehörige bei Veränderung automatisch verständigt werden. Technisch werde das durch ein Kamerasystem im Flurbereich realisiert, wie es die Universität Oldenburg derzeit testet. Beim kommerziellen Produkt PAUL (CIBEK GmbH, Limburgerhof) gibt es auf ähnliche Weise die Möglichkeit eines Aktivitätsmonitorings, welches durch Bewegungssensoren in der ganzen Wohnung realisiert wird.

\section{Roboter in der AAL: Was können sie (zusätzlich) leisten?}

Dietwig Lowet (Philipps, Niederlande), Leiter des EU-Projekts Florence sah die Vorteile der Robotik im Gegensatz zur klassischen AAL darin, dass die Kommunikation als natürlicher 
wahrgenommen werde, das System mobil sei und es ein stärkeres Gefühl der Anwesenheit vermittele. Zudem behauptete Lowet, dass die Überwachung mit einem Robotersystem im Gegensatz zu fest in der Wohnung installierten Kameras auf mehr Akzeptanz stoße, da sich intuitiv die Blickrichtung des Robotersystems erkennen lasse. Andreas Bley (MetraLabs, Ilmenau) wies darauf hin, dass Roboter nicht erst installiert werden müssten und damit ein Leihvertrieb ein wichtiges Geschäftsmodell werden könnte.

\section{Fünf Roboter für die AAL}

1. Das Florence-Projekt setzt, nach Lowet, auf roboterbasierte Fernassistenz durch Telepräsenzdienste, kameragestütztes Monitoring und kognitive Unterstützung durch angenehme Interaktion. Mit der Darstellung eines virtuellen Gesichts (Smiley) soll die Interaktionsbereitschaft mit dem System verstärkt werden. Der Roboter wird von Anfang an als Low-cost-System mit einem Verkaufspreis von 1.000 bis 2.000 Euro geplant.

2. Das Projekt ALIAS (Frank Wallhoff, Jade Hochschule, Oldenburg) hat eine ähnliche funktionale Ausrichtung, ist aber technisch und optisch hochwertiger ausgestattet und damit auch deutlich teurer (35.000 Euro). Um die Akzeptanz und Kommunikationsbereitschaft zu steigern (und dem System seine Fehler leichter nachzusehen), wurde ein Roboterkopf mit mechanischen Augen konzipiert, der das multifunktionale System als „Partner“" erscheinen lassen soll.

3. Der Care-O-Bot 3 (Birgit Graf, Fraunhofer IPA, Stuttgart) zeichnet sich dadurch aus, dass er im Gegensatz zu den anderen Roboterplattformen auch physische Aufgaben mit Hilfe eines mechanischen Armes erledigen kann bisher allerdings nur sehr langsam. Auch ist er nicht für ein genügend hohes Gewicht ausgelegt, um z. B. jemandem der gestürzt ist, aufhelfen zu können. Für die Bedienung sind, nach Graf, abgestufte Steuerungshierarchien für Pflegebedürftige, Pflegepersonal bzw. Angehörige geplant. Allgemein ist der Care-OBot 3 mit ca. 250.000 Euro bisher auch nur für Forschungszwecke geeignet. Eine Neu- vorstellung auf dem Kongress war ein deutlich kleineres und günstigeres System (unter 1.000 Euro), das spezialisiert auf Sturzerkennung automatisch eine Notfall-Videotelefonie-Verbindung zum Pflegepersonal schaltet.

4. Der Telepräsenz-Roboter Giraff lässt sich, wie Stephen von Rump (Giraff Technologies $\mathrm{AB}$, Schweden) selbst sagte, am besten als „Skype on wheels“ beschreiben. In diesem Projekt ist man so weit, dass mit der Markteinführung bereits begonnen wurde. Finanziert durch die Krankenkassen kommt der Roboter in Schweden bereits in einer städtischen Kommune (Västeras Stad) flächendeckend zum Einsatz. Von Rump gab aber zu, dass ohne die Fördergelder der EU das Projekt nicht weiterlaufen könne. Er wies ferner darauf hin, dass eine weitreichende Markteinführung aufgrund international stark divergierender Gesundheitssysteme und ihren Förderbedingungen schwierig sei.

5. Die Roboterplattform Nao des Projektes KSERA (Franz Werner, CEIT GmbH, Gilching) beansprucht den Vorteil, aufgrund der kleinen, menschenähnlichen Gestalt des Roboters sympathisch zu wirken und als Kommunikationspartner mit großer Akzeptanz rechnen zu können. Da Nao nicht über ein Display verfügt, wurde er mit einem Microbeamer erweitert, um Videotelefonie-Anwendungen zu realisieren. Zusätzlich lässt er sich als eine Art „Fitnesstrainer“ einsetzen, weil er physische Bewegungen vorführen kann.

\section{Machbarkeit und Kosten: Klassische AAL statt Robotik}

Bei der klassischen AAL stehen einfache und zuverlässige Lösungen im Vordergrund, die sich in Langzeittests bereits bewährt haben. Die Robotik dagegen bringt noch viele technische Probleme mit sich, wie u. a. das ALIAS-Projekt zeigte, etwa bei der autonomen Navigation, bei der Interaktion (z. B. bei der Sprachsteuerung) oder bei der Laufzeit (Akkulaufzeit und Aufladen). Auch die physischen Fähigkeiten sind für den praktischen Einsatz häufig ungenügend (zu wenig und zu langsame Bewegungsmöglichkeiten). Viele Robotersysteme sind auch noch zu teuer für den alltäglichen Einsatz und liegen weit über der in 
Nutzerbefragungen evaluierten Akzeptanzspanne von 1.000 bis 2.000 Euro. Die viel erhoffte Finanzierung durch die Krankenkassen ist dabei nur in Spezialfällen zu erwarten. AAL-Systeme bergen dagegen die Möglichkeit, von Wohnungsbaugesellschaften voreingebaut und später als Teil der Miete abbezahlt zu werden.

\section{Kritische Hinweise der Sozialwissenschaften}

Andreas Hein (Universität Oldenburg), Leiter des interdisziplinären Projekts OFFIS, äußerte mit Blick auf klassische AAL-Systeme Bedenken hinsichtlich der Privatheit, der möglichen Abhängigkeit vom System, sowie eines Zwangs zum normkonformen Verhalten im eigenen Zuhause.

Eine teilweise kritische Auseinandersetzung bezüglich mobiler Robotik-Systeme fand in der Session Robotik statt. Sebastian Glende (YOUSE $\mathrm{GmbH}$, Berlin) wies auf die weit verbreiteten Befürchtungen von Senioren gegenüber der möglichen Eigenständigkeit der Roboter und der Angst vor Überwachung hin. Katharina Scheibl (TU München) unterstrich die Wichtigkeit einer frühen Nutzereinbindung und berichtete, wie diese im ALIAS-Projekt in mehreren Evaluierungsphasen realisiert wurde.

Kolja Bopp, Manuel Dietrich und Knud Böhle (ITAS) setzten sich in ihrem Vortrag kritisch mit dem Leitbild „Artificial Companion“ bezüglich der Pflege auseinander. Dabei wurde zuerst das Leitbild "Artificial Companion“" aus Sicht der Informatik dargestellt. Sie versteht darunter ein technisches System, welches sich individuell auf Nutzer einstellt und als kompetenter und partnerschaftlicher Dienstleister erscheinen soll. Durch eine Expertenbefragung sei bestätigt worden, dass dieses Leitbild in der Technikentwicklung präsent sei. Auch vielen, auf dem Kongress vorgestellten Robotik-Projekten, liegt das Leitbild zugrunde: Zum Beispiel im Projekt ALIAS, in dem Roboter als soziale Akteure mit Persönlichkeit konzipiert werden, sei es paradigmatisch ausgeprägt. Bopp et al. stellten heraus, dass ein Leitbild auf verschiedenen Ebenen wirken könne. Eine Ebene betreffe die Funktion, Vorstellungen, die vom technisch, funktionalen Bereich ausgingen, in den Anwendungsbereich zu transportieren. Dieser Leitbildtransfer zwischen dem Bereich der
Informatik und dem der Pflege wurde im Beitrag als teilweise irreführend herausgestellt. Hierbei wurden Missverständnisse durch die CompanionMetapher und ein Täuschungspotenzial der Artefakte ausgemacht, die insbesondere mit Blick auf kognitiv eingeschränkte Nutzer ethische bedenklich seien. In der sich anschließenden Diskussion wurden anhand des Beispiels der Roboterrobbe Paro Aspekte der Übertragung von idealisierten Haustiereigenschaften auf technische Artefakte kritisch erörtert.

\section{Fazit}

Die Forschung und Entwicklung ist meist nicht vom Anwendungsbereich geleitet. Das heißt, die Impulse gehen selten von den Pflegewissenschaften oder den Bedarfen älterer Menschen aus. Fast alle Projekte arbeiten zwar mit kleinen Testgruppen, wobei diese aber meist schon mit einem fertigen Produkt konfrontiert werden. Dass sich die Pflege und das Leben zu Hause mit der vorgestellten Technik allerdings grundlegend ändern werden, ist in der nächsten Zeit nicht zu erwarten. Mit einer zwar langsamen aber stetigen Verbreitung, v. a. klassischer AAL-Systeme, ist indes zu rechnen.

Sozialwissenschaftliche und ethische Fragen, so der Eindruck von der Tagung, werden bei der Gestaltung der Technik noch zu wenig einbezogen, obwohl deren Berücksichtigung zentral für die oft angestrebte Markteinführung sein dürfte. Aus unserer Sicht wäre ein neuer, zusätzlicher Themenschwerpunkt für die jährlich stattfindende Tagung, der auf gleichberechtigte Weise Technik und Soziologie, Technik und Pflegewissenschaft zusammenbrächte und sich mit ,values in design" (Berücksichtigung von Wertvorstellungen in der Entwicklung) beschäftigte, ein Gewinn. 